\title{
Substrate texturing for homogeneous deposition of tilted c-axis AlN films for shear mode operation
}

\author{
M. Sierra-Zapata, M. Clement, T. Mirea, J. Olivares, and E. Iborra
}

\begin{abstract}
We propose two methods to improve the growth process of AIN films displaying the c-axis uniformly tilted, intended for the excitation of shear modes in acoustic resonators. The two methods aim at modifying the surface topography of the substrates in order to produce a family of parallel planes offering a suitable orientation for the subsequent growth of uniformly tilted AIN microcrystals. The first method is based on an ion milling process over rough substrates. Rough substrates, obtained by depositing a $\mathrm{W}$ layer on sputtered porous silicon oxide layers, are bombarded with a wide beam of $\mathrm{Ar}$ ions impinging on the sample surface at an angle of $65^{\circ}$. All treated surfaces lead to increased mean tilt angles of the subsequently grown AIN films, which in turn improves the coupling factor of the shear mode resonators. The second method consists in transferring the topography of a blazed diffraction grating to a photoresist layer spun over metal substrates. The photoresist pattern is then transferred to the substrates by a non- selective directional reactive ion etching process with $\mathrm{SF}_{6}$.
\end{abstract}

Keywords - BAW resonators; shear mode resonator; tilted grains $A I N$

\section{INTRODUCTION}

Gravimetric sensors based on electroacoustic resonators with surfaces functionalized to promote the binding of targeted species are good candidates for biological sensing in liquid environments. Compared with quartz crystal microbalances (QCMs), resonators based on AlN thin films offer the possibility of achieving greater sensitivities, since they can be operated at frequencies in the range of $\mathrm{GHz}$ by reducing the thickness of the piezoelectric film [1].

For in-liquid applications, it is advisable that gravimetric sensors based in bulk acoustic wave (BAW) resonators operate in the shear mode, which radiates less acoustic energy to the liquid medium than the longitudinal mode. To excite shear modes in piezoelectric AlN films a component of the electric field must be normal to the c-axis, to produce shear displacements by virtue of the $d_{15}$ piezoelectric coefficient. Two electrical configurations have been envisaged to achieve such excitation. The first one, called lateral excitation, is achieved by applying an electric field between two coplanar electrodes defined on top of a highly c-axis oriented film. This geometric arrangement would provide, according to previously reported works $[2,3]$, a straightforward excitation of the shear mode. The second configuration is based on the conventional excitation of AlN films using two electrodes placed on each side of the piezoelectric layer, but in this case, the AlN is made of microcrystals uniformly tilted with respect to the normal of the film, and this to the electric field [4]. This solution has proven to be far more effective than the lateral excitation [5]. It has been reported that tilt angles ranging from $20^{\circ}$ to $45^{\circ}$ provide the greatest values of the electromechanical coupling factor for the shear mode $\left(k_{\mathrm{s}}^{2}\right)$ [6]. Among the different techniques developed to achieve AlN films with tilted grains [7-9], off-axis sputter deposition over rough under-layers turns out to be the most effective [8]. In these kind of substrates, when the flux of atoms impinging on the different inclines is uniform, AlN grains tend to grow normally to the slopes in equal number in all directions, giving rise to a randomlyoriented AlN layer with averaged null piezoelectric response. However, if an impinging direction is encouraged (e.g. by displacing the substrate with respect to the target axis), the crystals will grow faster on the inclines most exposed to the atom flux and their mean tilt angle, normal to those planes, will be more uniform [9].

Two possible approaches can be used for controlling the uniformity and characteristics of these inclines. The first consists in carefully controlling the roughness of layers of the device that are closer to the active AlN film, either the bottom electrode or the uppermost layer of the acoustic reflector, in the case of solidly mounted resonators (SMRs). The second method involves the addition of a polycrystalline seed layer showing a controlled preferred orientation and exhibiting facets with the desired tilt angle $[4,10]$. Both methods suffer from a significant lack of homogeneity in the basal orientation of the inclines, leading to some dispersion in the direction of the AlN microcrystals.

The aim of this paper is investigate new procedures to homogenizing the orientation of the substrate inclines for the subsequent growth of AlN films with homogenously tilted grains. We propose two approaches to achieve this goal. The first one, hereafter "sculpturing", consist in pre-treating the substrates by Ar-ion milling at incident angles close to $65^{\circ}$ in order to sculpture the roughness of their surface before AlN deposition. The second approach, hereafter "nanoimprinting", involves nanoimprinting a photoresist layer spun over the bottom electrode of the SMR and etching it subsequently 
through a directional and poorly selective reactive ion etch (RIE) process, in order to transfer a saw-tooth-like profile to the substrate surface.

\section{METHODS}

The test substrates were $2 \times 2 \mathrm{~cm}^{2}$ silicon dies covered with a $\lambda / 4$ acoustic reflectors made of alternating $\mathrm{SiO}_{2}$ and $\mathrm{Mo}$ films as low and high acoustic impedance layers, respectively. The targeted resonant frequency was around $2.5 \mathrm{GHz}$ for the longitudinal mode and $1.4 \mathrm{GHz}$ for shear mode. The uppermost $\mathrm{SiO}_{2}$ layer of the reflector consisted in a sputtered porous layer displaying a mean roughness of around $4 \mathrm{~nm}$. As bottom electrode we used sputtered tungsten (W) films or evaporated iridium ( $\mathrm{Ir}$ ) films.

Before AlN deposition, some samples were subjected to a roughness sculpturing process. These samples were bombarded by ion milling with an extended $100 \mathrm{~mm}$ in-diameter Ar-ion beam produced by a Gencoa IM75 ion gun of variable ion energy and current. Other samples were subjected to the nanoimprinting process. First, they were covered with a photoresist layer, which was subsequently nanoimprinted on the center with a PDMS replica of a $12 \times 12 \mathrm{~mm}^{2}, 1200$ lines/mm diffraction grating having a blaze angle of $26^{\circ}$. The samples were then etched by $\mathrm{CHF}_{3} / \mathrm{SF}_{6}$-based reactive ion etching (RIE) process adjusted to reduce the etch selectivity between the photoresist and the underlying substrate. This method allowed us to transfer the imprinted profile to the $\mathrm{W}$ bottom electrode or the $\mathrm{SiO}_{2}$ uppermost layer of the SMR.

The AlN layers films were then sputtered in ultra-high vacuum system from a $150 \mathrm{~mm}$ in-diameter $\mathrm{Al}$ target in $\mathrm{Ar} / \mathrm{N}_{2}$ (40:60) mixtures. The substrates were displaced $5 \mathrm{~cm}$ from the center of the substrate holder, placed $5.8 \mathrm{~cm}$ apart from the target. The tilt angle of AlN grains was routinely derived from the position of the quasi-LO IR mode in the infrared reflectance spectra measured by Fourier transform IR spectroscopy (FTIR), using the method described in [11, 12]. Some samples were also assessed by measuring the XRD pole figure of the $(00 \cdot 2)$ reflection. Both measuring methods yielded similar values of the c-axis tilt angle.

BAW resonators were produced by adding a Mo top electrode to the whole structure. They were characterized by deriving their electrical impedance from the measurement of the reflection coefficient $\left(\mathrm{S}_{11}\right)$ performed with a network analyzer between $100 \mathrm{MHz}$ and $5 \mathrm{GHz}$.

\section{RESULTS AND DISCUSSION}

\section{A. Texturing the roughness of the substrate}

Opposite to the growth of highly $c$-axis oriented AlN films, which requires polishing the surface of the acoustic mirrors, tilted AlN films can be achieved by taking advantage of the native roughness of the substrates combined with an off-axis sputter deposition. However, if this surface is not previously conditioned, the resulting AIN film displays a significant dispersion in the tilt angle. Trying to improve the uniformity of the inherently rough surface of an acoustic mirror, resulting from the successive growth of the different composing layers, we decided sculpture the surface of the $\mathrm{W}$ bottom electrodes of several devices by ion milling them at $65^{\circ}$ with respect to the surface normal at different ion energies and currents. During the process a part of each sample was intentionally masked with a glass plate so that the subsequent AlN film grew simultaneously on treated and not-treated areas. After the ion milling the samples were immediately loaded in the sputtering chamber for the off-axis AlN sputter process. The position of the Al-LO peak in the IR reflectance spectra measured in the sculptured part of the samples was systematically shifted toward greater wavenumbers, revealing that the sculpturing process tended to increase the tilt angle of the samples, to a greater or lesser extent.

Figure 1 shows the tilt angle derived from FTIR measurements of AlN films grown on W electrodes sculpted for 20 minutes under different experimental conditions. It is important to note that the data of figure 1 correspond to sample dies coming from the same $100 \mathrm{~mm}$ silicon wafer covered with a five-layer $\mathrm{SiO}_{2} / \mathrm{Mo}$-based acoustic reflector. This is so because samples coming from different substrates give rise to strong variations in the tilt angle, due to the uncontrolled roughness profiles of the acoustic reflectors from run to run. Results of figure 1 reveal that there is a threshold voltage (1050 V) above which the ion-milling process has a significant effect in the surface conditioning that promotes the growth of more tilted AlN films. Additionally, for voltages values above the threshold, lower ion currents induce greater tilt angles, which suggests that a too high bombardment may produce a smoothing of the rough surface.

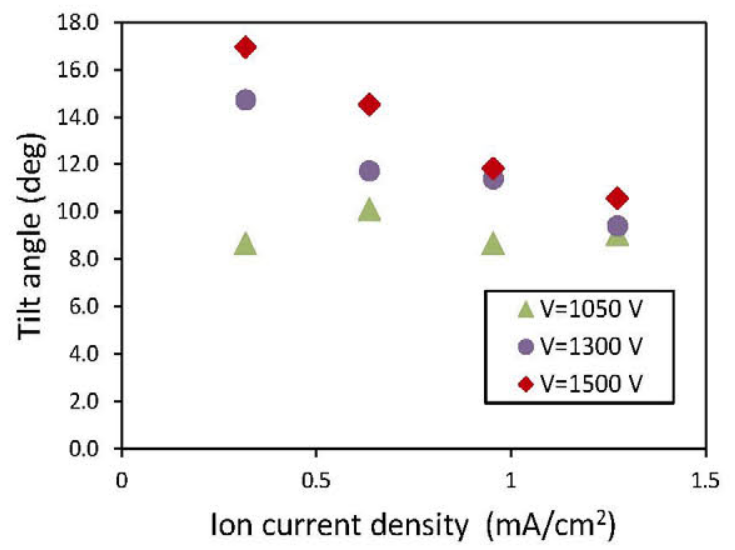

Fig. 1. Tilt angle of AIN films grown over ion milled W substrates as a function of the ion current density and the Ar ion acelerating voltage

However, despite the evident increase of the tilt angle, the electric characterization of the resonators did not show a clear improvement in the performance of the shear modes, being impossible to find a trend between tilt angle and electromechanical coupling factor $k_{s}^{2}$. All the results obtained in a large number of samples treated under different conditions suggested that the uncontrolled roughness profiles of the reflectors are hardly homogenized by an ion-milling process. Moreover, the lower values of $k_{s}^{2}$ obtained in some samples after the ion milling treatment pointed out that, in some cases, the latter might even induce damage in the sample, leading to AlN films of lower piezoelectric activity. 


\section{B. Transfer of slopes by nanoimprint and RIE}

To achieve greater tilt angles and improve uniformity and reproducibility of the engineered surfaces, we developed a twostep profile transfer method based on, first, nanoimprinting a saw-tooth profile on a thin photoresist layer (400 $\mathrm{nm}$ thick) using a stamp made of PDMS, and then transferring the profile to the substrate. The actual master for the stamp was a 1200 lines $/ \mathrm{mm}$ ruled diffraction grating with a blaze angle of $26^{\circ}$. The transfer method was a RIE process based on an $\mathrm{SF}_{6}$ plasma for $\mathrm{W}$ layers and on $\mathrm{CHF}_{3}$ and $\mathrm{SF}_{6}$ mixtures for $\mathrm{SiO}_{2}$ layers. The etching processes were tuned to obtain the same etch rate for the resist and the layer underneath. Figure 2 shows a scheme of the etching process at different moments.

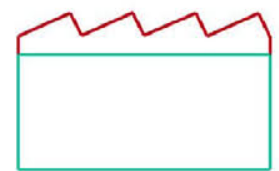

(1)

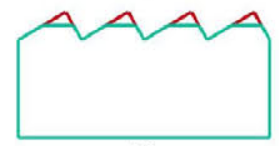

(3)

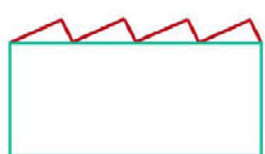

(2)

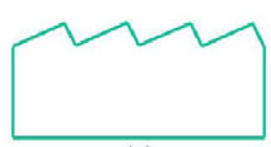

(4)
Fig. 2. Scheme of the transfer etching process. (1) starting profile; (2) moment just before starting substarte etching; (3) same etch rate for resist and substrate maintain the geometry; (4) end of the process.

Similar profiles of the surface topography were obtained when transferring the pattern directly to the $\mathrm{W}$ bottom electrode or when transferring the pattern to the $\mathrm{SiO}_{2}$ outermost layer of the acoustic reflector before the growth of the $\mathrm{W}$ electrode. Fig. 3 shows two AFM 3D images of the topography of the $\mathrm{W}$ top electrode. Picture a) corresponds to a W substrate directly subjected to the profile transfer process and picture b) to a $\mathrm{W}$ layer grown on top of a nanoimprinted $\mathrm{SiO}_{2}$ layer.
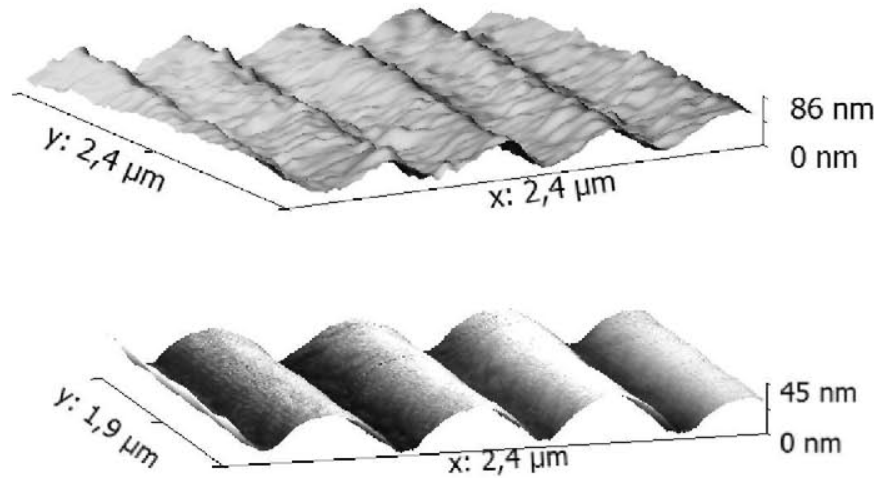

Fig. 3. AFM image of the surface of a) $\mathrm{W}$ film patterned through the twostep nanoimprinting process and b) W film deposited on a textured $\mathrm{SiO}_{2}$ layer.

AlN films grown of top of $\mathrm{W}$ films textured through the nano-imprinting process tended to grow normal to the substrates, regardless of the surface topography. This is due to the fact that AlN microcrystals grow with the $(00 \cdot 2)$ planes parallel to the (110) planes of the $\mathrm{W}$ grains, which always grow parallel to the surface when deposited by sputtering, regardless of the sputtering conditions. In fact, we varied the W sputter parameters and sample position in the substrate holder to investigate the effect of the directionality of the sputter process but we achieved always the same results. To solve this problem, we had to introduce a decoupling layer to prevent AlN grains from growing with the c-axis normal to the (110) planes of $\mathrm{W}$. We tried with $\mathrm{SiO}_{2}$ (without success) and with AlN thin layers sputtered under low energy conditions -low power $(600 \mathrm{~W})$ and high pressure ( $5 \mathrm{mTorr})$ - to promote the growth of randomly oriented AlN microcrystals. This way we succeed in "erasing" the memory of the surface and growing AlN films with the c-axis normal to the $\mathrm{W}$ layer inclines, as shown in the sketches of figure 4 . The thickness of this AlN thin layer played an important role on the tilt angle of the subsequent AlN films as shown in figure 5.

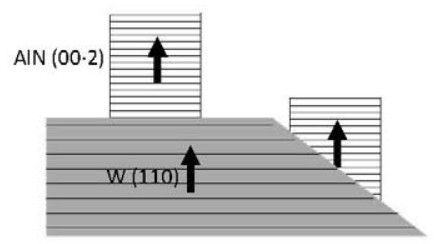

a)

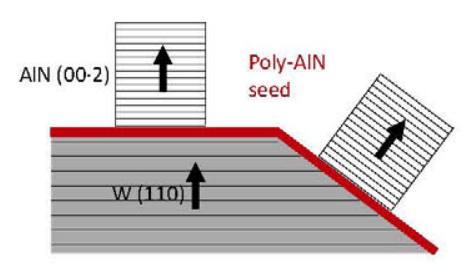

b)
Fig. 4. Sketch showing the mechanism that prevents the AlN grains from growing normally to the surface on engineered $W$ films. a) normal growth on $\mathrm{W}$ inclines and $\mathrm{b}$ ) growth on $\mathrm{W}$ slopes afer erasing the surface memory via a poly-AlN layer

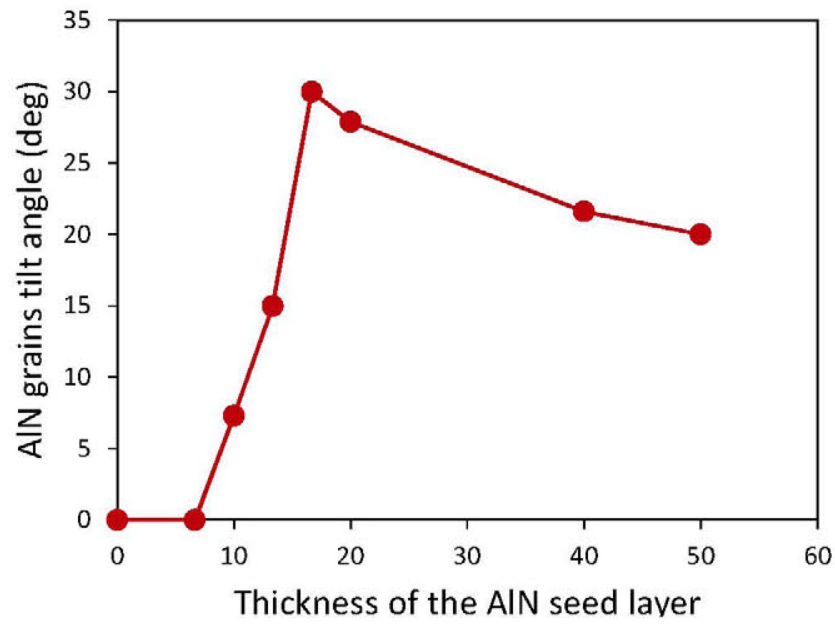

Fig. 5. Tilt angle of the piezoelectrc AlN films grown of top of engineered surfaces as a funtion of the thickness of the poly-Aln film.

With the help of the poly-AlN decoupling layer we were able to produce AlN films with uniformly inclined microcrystals with mean tilt angles close to $30^{\circ}$ from the normal, as shown in the XRD pole measurements of figure 6 . These plots reveal that there is clear improvement in the uniformity of the tilt angle after the conditioning of the surface topography, which is additionally shifted to higher values (around $30^{\circ}$ ). 

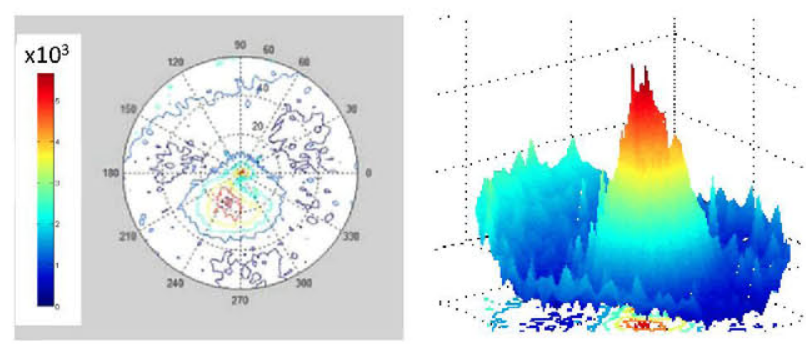

XRD pole measurements in non-imprinted areas
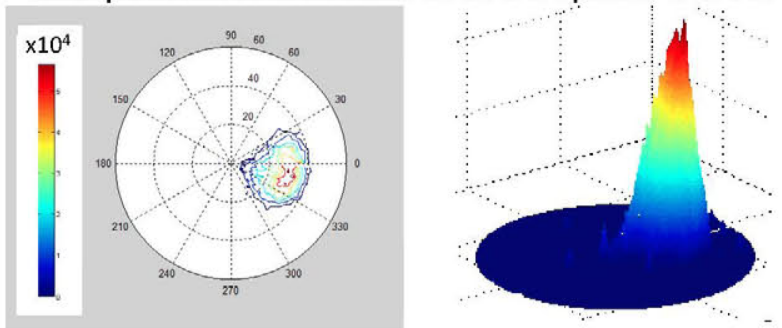

XRD pole measurements in imprinted areas

Fig. 6. Pole figure of the $(00 \cdot 2)$ AIN XRD reflection in non-imprinted and imprinted areas of a same sample.

Finally, we show in figure 7 the values of the electromechanical coupling factors achieved in the resonators subjected to surface conditioning. The shear mode resonators achieved by the two methods have coupling factors greater than those displayed by non-conditioned SMRs, reaching values close to $3 \%$, similar to those obtained only by adding a miss-oriented thicker AlN seed layer on smooth substrates [10].

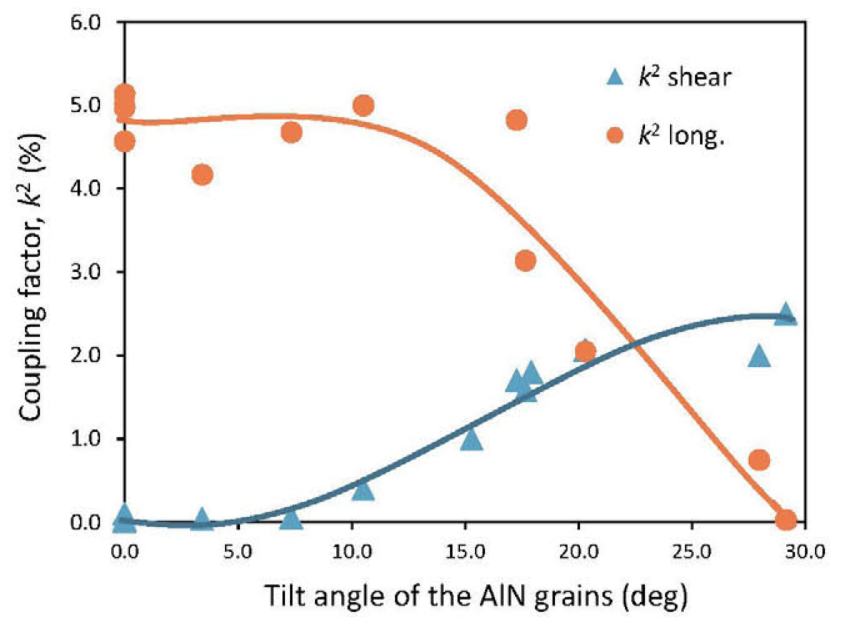

Fig. 7. Variation of the coupling factor of longitudinal and shear modes as a function of the AIN grain tilt angle.

\section{CONCLUSIOTNS}

We investigate two new methods to engineer the bottom electrodes of SMRs in order to achieve tooth saw-like profiles that promote the growth of tilted AlN films. The first method, based in texturing the inherent roughness of the acoustic mirror surface by ion milling it at high angles $\left(65^{\circ}\right)$, leads to an increase in the tilt angle of the grains of the subsequently grown AlN films, but does not improve their piezoelectric activity. The second method, based on sculpturing the surface of the bottom electrodes in a saw-tooth pattern throw a twostep nanoimprinting-RIE process does not promote by itself the growth of tilted films. The influence of the metal substrate crystallinity prevails over that of the substrate topography. A randomly oriented AlN seed layer prevents the AlN films from growing along the preferred orientation of the metallic surfaces. Films grown of imprinted samples display improved uniformity of the tilt angle, which reaches values of $30^{\circ}$.

\section{ACKNOWLEDGMENT}

This work was partially supported by Ministerio de Economía y Competitividad del Gobierno de España through project MAT2013-45957-R.

\section{REFERENCES}

[1] Mario DeMiguel-Ramos, Bárbara Díaz-Durán, José-Miguel Escolano, Mariano Barba, Teona Mirea, Jimena Olivares, Marta Clement, Enrique Iborra, "Gravimetric biosensor based on a $1.3 \mathrm{GHz}$ AlN shear-mode solidly mounted resonator". Sensors and Actuators B 239 (2017) 1282 1288. DOI: $10.1016 /$ j.snb.2016.09.079.

[2] E. Milyutin, S. Gentil, P. Muralt, Shear mode bulk acoustic wave resonator based on c-axis oriented AlN thin film, J. Appl. Phys. 104 (2008) 084508

[3] Da Chen, J. Wang, D. Li, Y. Xu, Z. Li, Solidly mounted resonators operated inthickness shear mode based on c-axis oriented AlN films, Sens. Actuators A 165 (2011) 379-384.

[4] G. Wingqvist, J. Bjurstr€om, A. Hellgren, and I. Katardjiev, Sens. Actuators, B 127(1), 248 (2007).

[5] M. Clement, E. Iborra, J. Olivares, M. DeMiguel-Ramos, T. Mirea, J. Sangrador. "On the effectiveness of lateral excitation of shear modes in AlN layered resonators" Ultrasonics 54 (2014) pp. 1504-1508; DOI: 10.1016/j.ultras.2014.04.014

[6] J. Bjurström, D. Rosén, I. Katardjiev, V. Yantchev, I. Petrov, Dependence of the Electromechanical Coupling on the Degree of Orientation of c-Textured Thin AlN Films, T. Ultrason. Ferr. Freq. Control 51(10) (2004) 1-7.

[7] T. Yanagitani and M Kiuchi, Control of in-plane and out-of-plane texture in shear mode piezoelectric $\mathrm{ZnO}$ films by ion-beam irradiation, Journal of Applied Physics 102 (2007) 044115.

[8] M. Moreira and J. Bjurström, Synthesis of c-tilted AlN films with a good tilt and thickness uniformity, Proceedings of IEEE Ultrasonics Symposium (2012) 1238-1241.

[9] J. Bjurstrom, G. Wingqvist and I. Katardjiev, Synthesis of textured thin piezoelectric AlN films with a nonzero $\mathrm{C}$-axis mean tilt for the fabrication of shear mode resonators, Transactions on Ultrasonics, Ferroelectrics and Frequency Control (2006) 2095-2100.

[10] M. DeMiguel-Ramos, T. Mirea, M. Clement, J. Olivares, J. Sangrador and E. Iborra, "Optimized tilted c-axis AlN films for improved operation of shear mode resonators" Thin Solid Films 590 (2015) 219-223.

[11] E. Iborra, M. Clement, L. Vergara, A. Sanz-Hervás, J. Olivares, J. Sangrador "Dependence of the IR reflectance LO absorption bands on the crystalline texture of AlN films" Applied Physics Letters 88 (2006) pp. 231901.1-3

[12] J. Olivares, S. González-Castilla, M. Clement, A. Sanz-Hervás, L. Vergara, J. Sangrador, E. Iborra "Combined assessment of piezoelectric AlN films using X-ray diffraction, infrared absorption and atomic force microscopy" Diamond and Related Materials. Vol. 16 (2007) pp.14211424. 